\title{
Reports of Envenomation by Brown Recluse Spiders Exceed Verified Specimens of Loxosceles Spiders in South Carolina
}

\author{
Ivar L. Frithsen MD, MS, Richard S. Vetter, MS, and Ian C. Stocks, MS
}

Purpose: To determine whether the number of brown recluse spider bites diagnosed by South Carolina physicians coincides with evidence of brown recluse spiders found in the state.

Methods: Brown recluse spider bite diagnosis data were extracted from 1990 and 2004 surveys of South Carolina physicians. This was compared with the known historical evidence of brown recluse spiders collected in South Carolina and derived from various sources, including state agencies, arachnologists, and museum specimens.

Results: South Carolina physicians diagnosed 478 brown recluse spider bites in 1990 and 738 in 2004. Dating to 1953, 44 brown recluse spider specimens have been verified from 6 locations in South Carolina.

Discussion: The number of brown recluse bites reportedly diagnosed in South Carolina greatly outnumbers the verified brown recluse specimens that have been collected in the state. The pattern of bite diagnoses outnumbering verified brown recluse specimens has been reported in other areas outside of this spider's known endemic range. (J Am Board Fam Med 2007;20:483-488.)

The range of the brown recluse spider, Loxosceles reclusa, is restricted to a specific portion of the southeastern and central United States, which potentially includes the westernmost tip of South Carolina ${ }^{1}$ (Figure 1). Despite this limited distribution, necrotic skin lesions have been attributed to loxoscelism (bites by Loxosceles spiders) throughout the continental United States, Canada, and Alas$\mathrm{ka}^{2-4}$ In many areas where bites by brown recluse

This article was externally peer reviewed.

Submitted 21 December 2006; revised 8 June 2007; accepted 13 June 2007.

From the Department of Family Medicine, Medical University of South Carolina, Charleston (ILF); the Department of Entomology, University of California, Riverside (RSV); the Biology Division, San Bernardino County Museum, Redlands, CA (RSV); and the Department of Entomology, Soils, and Plant Sciences, Clemson University, Clemson, SC (ICS).

Funding: Supported in part by grant 1D14 HP 00161 from the Health Resources and Services Administration (ILF). Supported in part by South Carolina Public Services Activities, Division of Regulatory Services, Plant Problem Clinic, and USDA Cooperative State Research, Education, and Extension Service, grant no. 2002-30001-12082. Southern Plant Diagnostic Network (SPDN) (ICS).

Conflict of interest: none declared.

Corresponding author: Ivar Frithsen, Department of Family Medicine, Medical University of South Carolina, 295 Calhoun Street, Charleston, SC 29425 (E-mail: frithse@musc.edu). spiders are commonly reported, verified specimens are rare, no current populations are known to exist, and, in some cases, no Loxosceles spiders have ever been found. ${ }^{2-5}$ This disparity between large numbers of reported loxoscelism cases versus few verified brown recluse specimens in areas outside of their native range signifies that this condition is over diagnosed in many parts of the country.

There are many alternate causes of skin lesions that have been mistaken for brown recluse spider bites, ${ }^{1}$ including methicillin-resistant Staphylococcus aureus (MRSA). MRSA is a rapidly emerging infectious disease threat in many parts of the United States. ${ }^{6-9}$ A recent study of patients with skin and soft-tissue infections presenting to 11 different emergency departments noted that MRSA was isolated from $59 \%$ of patients. This led to the conclusion that MRSA is now the most common cause of skin and soft tissue infections in the cities studied. ${ }^{10}$ The prevalence of MRSA was noted to be $68 \%$ in Charlotte, North Carolina, and $72 \%$ in Atlanta, Georgia, during August 2004. ${ }^{10}$ Studies have shown that patients with MRSA infections frequently present to their physicians with a complaint of spider bite. ${ }^{10,11}$ The severe pain often experienced by patients with MRSA skin infections is 


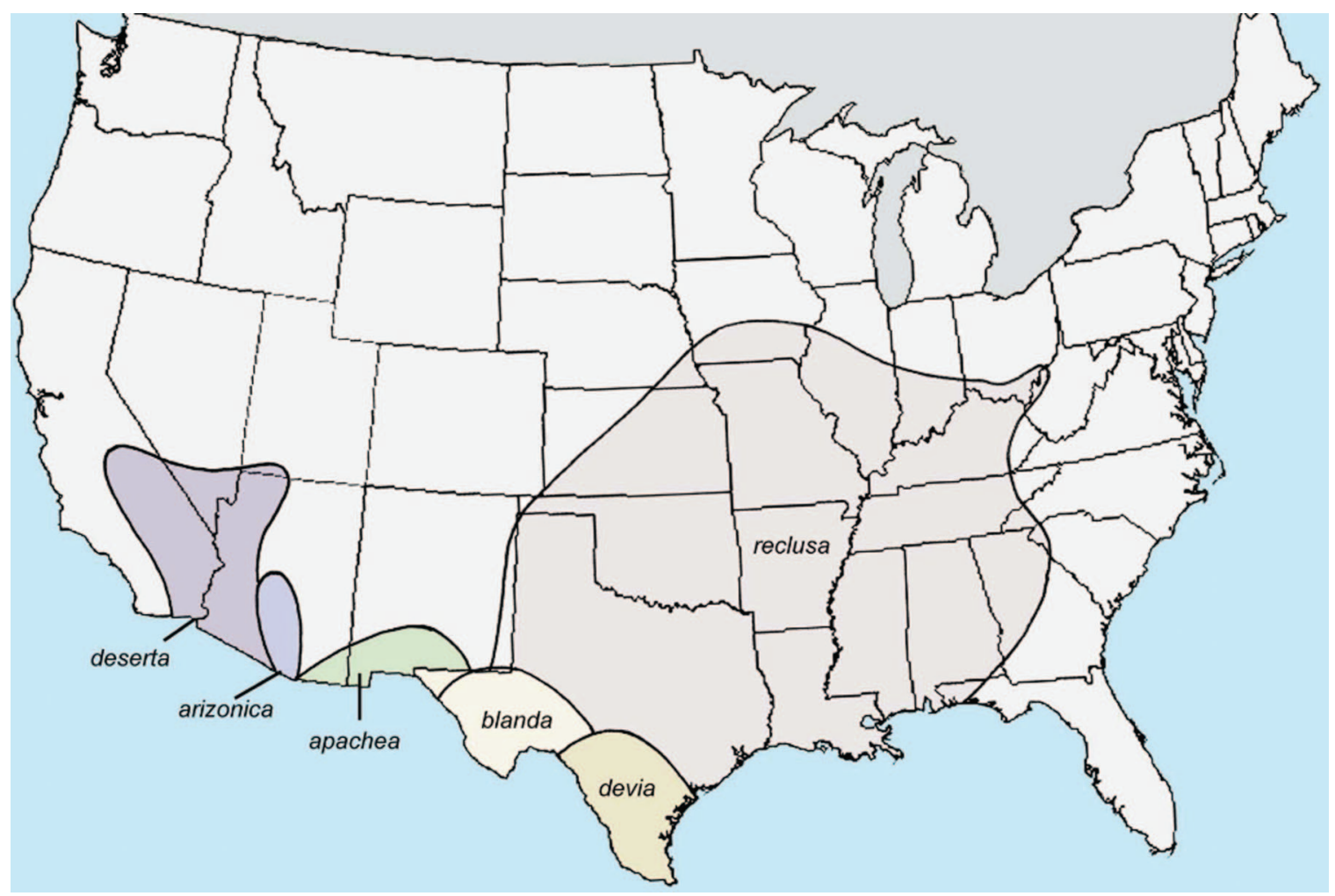

Figure 1. Geographic distribution throughout the United States of verified widespread populations of 6 native Loxosceles species. Reprinted with permission of the Massachusetts Medical Society.

probably the reason they think they have been bitten by a spider. ${ }^{12}$ Therefore, in areas where brown recluse bites are commonly diagnosed but brown recluse spiders are rare, it is likely that MRSA infections are being missed. This would be especially true where MRSA prevalence is known to be high, making it important to examine spider bite data in these areas.

Although there are no published reports indicating the prevalence of MRSA in South Carolina, based on the numbers noted above from neighboring states it is likely that MRSA infections are common in South Carolina. Another reason for studying this issue is that the westernmost tip of South Carolina is on or just beyond the natural range of the brown recluse spider. In addition, data concerning the number of physician-diagnosed brown recluse bites is available from surveys of South Carolina physicians that were conducted in 1990 and 2004. ${ }^{13,14}$ Finally, there are several available sources for determining the distribution of brown recluse spiders in South Carolina, including accessible collections from national and state museums and information obtained from Clemson University entomologists. The objective of this study was therefore to determine whether the known distribution of brown recluse spiders in South Carolina corresponds to statewide reports of loxoscelism. Information compiled here would be useful for physicians anywhere outside of the endemic brown recluse range.

\section{Methods}

\section{Physician Surveys}

To determine the extent of physician-diagnosed brown recluse spider bites in South Carolina, data were tabulated from 2 statewide surveys of physicians. $^{13,14}$ The most recent survey reported the number of bites diagnosed by primary care physicians during 2004. This survey was mailed to 2789 South Carolina physicians identified as family practitioners, internists, and pediatricians from the $\mathrm{Di}$ rectory of the State Board of Medical Examiners. All full-time medical school faculty members were excluded. The surveys were sent in February 2005 and returned via facsimile or mail between March and July 2005. Physicians were asked to report the total number of injuries from various arthropods (spiders, ticks, and fire ants) they had diagnosed 
during 2004 and asked to include information about brown recluse bites. Further details of this survey, including the full survey instrument, have been previously published. ${ }^{13}$

Data on diagnoses of brown recluse bites were also extracted from a previously published 1990 study. This study was similar to the one described above, with the exception that it was not limited to primary care physicians. Instead it was mailed to 2366 physicians in practices identified in the Directory of the State Board of Medical Examiners as family practice, internal medicine, pediatrics, general practice, emergency medicine, and occupational medicine. In early 1991 physicians were sent a survey asking them to tabulate the total number of patients they had seen in 1990 with a diagnosis of a brown recluse bite. Further details of this study have been previously published. ${ }^{14}$

\section{Arachnological Evidence}

To determine the numbers and locations of brown recluse spiders that are known to have been collected in South Carolina, information was obtained from various state agencies. Private citizens, pest control operators, and physicians from South Carolina submit spiders to Clemson University, the South Carolina Forestry Commission, and the South Carolina Department of Health and Environmental Control for expert identification, often under the suspicion that the specimens may be brown recluses. Officials at each of these agencies were contacted to determine whether there are either written accounts or personal recollections of brown recluse submissions from any source. Additional information was provided by communication with arachnologists and entomologists who had personal experience in the state.

For over 80 years, the Clemson University Arthropod Collection has been a major repository for spiders and other arthropods collected from across South Carolina; this resource was examined for brown recluse specimens. In addition, several major American museums contain depositories of spiders collected and submitted from various sources throughout North America, including South Carolina. Loxosceles specimens collected from South Carolina were requested from the American $\mathrm{Mu}-$ seum of Natural History (New York, NY); the National Museum of Natural History (Smithsonian, Washington DC); the Field Museum of Natural History (Chicago, IL); the California Academy

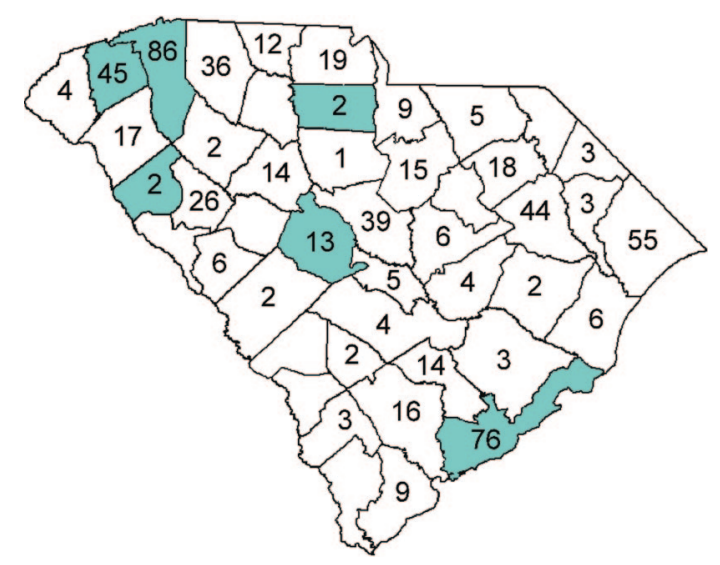

Figure 2. The number of brown recluse bites reported by primary care physicians in 2004 , by county.

of Sciences (San Francisco, CA); the Denver $\mathrm{Mu}$ seum of Nature and Science (Denver, CO); the Florida State Collection of Arthropods (Gainesville, FL); the Burke Museum of Natural History (Seattle, WA); and the Clemson University Arthropod Museum (Clemson, SC). Loxosceles spiders were examined from any of these museums that were found to contain specimens collected in South Carolina. An additional source of arachnological evidence was an annotated checklist providing information on common South Carolina spiders; this included the names of species found in the state and the locations where specimens have been collected. ${ }^{15}$

\section{Results}

\section{Physician Surveys}

For the 2004 survey, there were 514 total responses (overall response rate, 19\%). At least one brown recluse bite diagnosis was reported by $44 \%$ ( $\mathrm{n}=$ 226) of respondents during 2004; a total of 738 bites were reported. The mean number of bites reported per provider who diagnosed at least 1 case of loxoscelism in 2004 was $3.3 .^{13}$ The number of bites reported by county is included as Figure 2 . For the 1990 survey, there were 940 responses (overall response rate, $42 \%$ ). At least one brown recluse bite diagnosis was reported by $23 \%$ ( $\mathrm{n}=$ 217) of respondents during 1990; a total of 478 bites were reported. The mean number of bites reported per provider who diagnosed at least 1 case of loxoscelism in 1990 was 2.2. ${ }^{14}$

\section{Arachnological Evidence}

A total of 44 brown recluse spiders have been documented or reported in 6 of South Carolina's 46 
counties, with all finds collected at one location per county. Figure 2 shows the counties where verified brown recluse specimens were collected (shaded blue). The Clemson University Arthropod Museum contains 8 brown recluse specimens (from a single apartment) collected in South Carolina out of 8800 spider specimens dating from 1925. The Field Museum in Chicago contains 13 brown recluse spiders that were collected from 2 locations in South Carolina. None of the other museums reported any brown recluse specimens from South Carolina in their collections. An annotated publication on South Carolina spiders lists 23 brown recluses collected from 3 different locations in the state. ${ }^{15}$ No officials from the state agencies that routinely receive spider specimens for identification could recall a brown recluse submission and no written records of brown recluse specimens were noted. None of these specimens were known to be involved in envenomations.

\section{Discussion}

The numbers of physicians responding to both the 1990 and 2004 surveys represent only a fraction of the physicians practicing in South Carolina; therefore it is likely that many more cases of loxoscelism are diagnosed there. Yet in both years, the numbers of brown recluse bites reported by South Carolina physicians were more than was reported to the American Association of Poison Control Centers for the entire country during the same time periods. ${ }^{16,17}$ However, the American Association of Poison Control Centers data includes more than just physician-diagnosed bites and data on spider bites should be cautiously interpreted, ${ }^{18}$ it is the only national data available on reported loxoscelism. In 2003 a regional medical journal article encouraged skepticism when diagnosing brown recluse bites in nonendemic areas. ${ }^{19}$ However, increases were noted from 1990 to 2004 in both the percentage and the number of South Carolina physicians reporting loxoscelism. The average number of brown recluse bites reported by physicians diagnosing at least 1 case also increased from 1990 to 2004.

The number of verified South Carolina specimens of $L$. reclusa spiders is minimal when compared with the population sizes found within their endemic range. In the central portion of the known habitat range of the brown recluse (ie, Kansas,
Oklahoma, Missouri, Arkansas), multiple specimens have been easily collected from single locations. For example, researchers documented over 2000 brown recluse spiders in a single Kansas home over a 6 month period. ${ }^{20}$ An additional study in Kansas showed that 22 of 25 homes had brown recluses; traps set out captured a mean of $83.5 \pm$ 114.9 per house (range, $1-526) .{ }^{21}$ Because no similar discoveries have been reported in South Carolina, it is unlikely that the brown recluse exists here in high numbers. It is also noteworthy that, in their endemic range, brown recluses can be collected from both natural habitats (eg, rock outcrops) and from man-made structures like homes and buildings. In contrast, brown recluses in South Carolina have been collected exclusively from man-made structures. This observation supports the claim that brown recluse populations in South Carolina are the result of introductions; therefore these populations are localized and unlikely to spread by dispersal through natural habitats. The lack of brown recluse spider evidence is consistent with a 1944 study where no Loxosceles specimens were noted among 350 species of spiders collected from Georgia and South Carolina and identified by arachnologists. ${ }^{22}$ However, a limitation of our study is that all spider species located in a state are not necessarily included in museum collections.

It is possible that private citizens have found brown recluse spiders in South Carolina that were not submitted to state agencies for identification and that collections of spiders in museums may not accurately reflect the actual population range of the brown recluse spider on the southeastern margin of its distribution. However, the arachnological evidence from the various sources presented here is the best estimate of the population distribution that can be assembled; these museum collections and publications represent the collecting efforts of many scientists and the general public over decades. It is highly unlikely that an additional extensive statewide collection effort would significantly alter the arachnological picture presented here. Another limitation of this study is that the physician diagnosis data are based on recall, which is known to be unreliable on an individual basis; however, recall can be an efficient means of obtaining population level data. Despite these limitations, the findings of this study should lead physicians to be cautious in making a presumptive diagnosis of brown recluse bite in South Carolina. 
Several authors have noted broad differential diagnoses to be considered in cases of suspected loxoscelism including several conditions commonly seen in South Carolina, ${ }^{23-25}$ such as Rocky Mountain spotted fever, Lyme disease, herpes zoster, and diabetic ulcers. However, considering the recent emergence of MRSA as a skin and soft tissue pathogen, MRSA infections may represent a larger portion of suspected brown recluse bites in South Carolina than previously suspected. Physicians should maintain a high level of suspicion for MRSA in any patient with a skin or soft tissue infection, but especially if the patient presents with a complaint of spider bite. A common misconception is that brown recluse spiders can be found anywhere in North America because they could be inadvertently transported during commerce, but the arachnological evidence shows that Loxosceles spiders are rare outside endemic areas. ${ }^{5}$ Because brown recluse bites are rare even in endemic areas, short-term travel to such regions should likewise not cause consideration of loxoscelism diagnoses without sufficient incriminating evidence. In nonendemic Loxosceles areas, such as South Carolina, physicians should only diagnose brown recluse spider bites if recluse spider involvement can be definitively proven. Spider specimens should be obtained from patients whenever possible and submitted for expert identification by arachnologists (information on how to submit a sample for identification is included below).

\section{Conclusions}

This study adds South Carolina to the list of locations where loxoscelism reports outnumber historically verified specimens of Loxosceles spiders. ${ }^{2-4}$ The erroneous diagnosis of loxoscelism in South Carolina and other areas outside of this spider's known range may delay treatment of other conditions such as MRSA infection. Further research into this issue is necessary, including prospective studies designed to determine the true etiology of suspected spider bites. Isbister summarized the issue well by stating that, "The myth of necrotic arachnidism must be debunked by accurately defining the effects of definite spider bites and simultaneously investigating necrotic ulcers to determine a cause. $" 26$

\section{Spider Submission}

Submit spiders for identification to: Richard S. Vetter, MS, Department of Entomology, 3401 Watkins Drive, University of California, Riverside, Riverside, CA 92521, or Ian C. Stocks, MS, Department of Entomology, Soils, and Plant Sciences, Clemson University, Clemson, SC 29634

Live or dead spiders can be sent in an unbreakable container with a tightly secured lid. A small amount of crumpled paper towels placed in the container and secured will prevent excessive damage to the spider during shipping. It is not necessary to provide food, water, or air holes for live spiders.

We would like to acknowledge William M. Simpson, Jr, MD, and Arch Mainous, $\mathrm{PhD}$, for their assistance in the preparation of this manuscript.

\section{References}

1. Swanson DL, Vetter RS. Bites of brown recluse spiders and suspected necrotic arachnidism. N Engl J Med 2005;352:700-7.

2. Vetter RS, Cushing PE, Crawford RL, Royce LA. Diagnoses of brown recluse spider bites (loxoscelism) greatly outnumber actual verifications of the spider in four western American states. Toxicon 2003;42: 413-8.

3. Vetter RS, Edwards GB, James LF. Reports of envenomation by brown recluse spiders (Araneae: Sicariidae) outnumber verifications of Loxosceles spiders in Florida. J Med Entomol 2004;41:593-7.

4. Bennett RG, Vetter RS. An approach to spider bites: erroneous attribution of dermonecrotic lesions to brown recluse or hobo spider bites in Canada. Can Fam Physician 2004;50:1098-101.

5. Vetter RS. Arachnids submitted as suspected brown recluse spiders (Araneae: Sicariidae): Loxosceles spiders are virtually restricted to their known distributions but are perceived to exist throughout the United States. J Med Entomol 2005;42:512-21.

6. Eady E, Cove J. Staphylococcal resistance revisited: community-acquired methicillin resistant Stapbylococcus aureus-an emerging problem for the management of skin and soft tissue infections. Curr Opin Infect Dis 2003;16:103-24.

7. Herold B, Immergluck L, Maranan M, et al. Community-acquired methicillin-resistant Staphylococcus aureus in children with no identified predisposing risk. JAMA 1998;279:593-8.

8. Crum N, Lee R, Thornton S, et al. Fifteen-year study of the changing epidemiology of methicillinresistant Staphylococcus aureus. Am J Med 2006;119: 943-51.

9. Purcell K, Fergie J. Exponential increase in commu- 
nity-acquired methicillin-resistant Staphylococcus aureus infections in South Texas children. Pediatr Infect Dis J 2001;20:860-3.

10. Moran GJ, Krishnadasan A, Gorwitz RJ, et al. Methicillin-resistant $S$. aureus infections among patients in the emergency room. N Engl J Med 2006; 355:666-74.

11. Dominguez TJ. It's not a spider bite, it's community-acquired methicillin-resistant Staphylococcus aureus. J Am Board Fam Pract 2004;17:220-6.

12. Rutecki GW. Methicillin-resistant staph on the riseand on the loose. Consultantlive. 2007;47:533.

13. Frithsen IL, Simpson WM. 2004 South Carolina physician survey of tick, spider and fire ant morbidity. J SC Med Assoc 2006;102:153-8.

14. Schumann S, Caldwell S. 1990 South Carolina physician survey of tick, spider and fire ant morbidity. J SC Med Assoc 1991;87:429-432.

15. Gaddy LL, Morse JC. Common spiders of South Carolina with an annotated checklist. SC Agric Experiment Station Tech Bull 1985;1094:182.

16. Watson WA, Litovitz TL, Rodgers GC Jr, et al. 2004 annual report of the American Association of Poison Control Centers Toxic Exposure Surveillance System. Am J Emerg Med 2005;23:589-666.

17. Litovitz T, Bailey KM, Schmitz BF, Holm KC, Klein-Schwartz W. 1990 annual report of the American Association of Poison Control Centers Toxic Exposure Surveillance System. Am J Emerg Med 1991;9:461-509.
18. Vetter RS, Furbee RB. Caveats in interpreting poison control centre data in spider bite epidemiology studies. Public Health 2006;120:179-81.

19. Wendell RP. Brown recluse spiders: a review to help guide physicians in nonendemic areas. South Med J 2003;96:486-90.

20. Vetter RS, Barger DK. An infestation of 2,055 brown recluse spiders (Araneae: Sicariidae) and no envenomations in a Kansas home: implications for bite diagnoses in non-endemic areas. J Med Entomol 2002;39:948-51.

21. Sandidge J. Predation by cosmopolitan spiders upon the medically significant pest species Loxosceles reclusa (Araneae: Sicariidae): limited possibilities for biological control. J Econ Entomol 2004;97:230-4.

22. Chamberlin RV, Ivie W. Spiders of the Georgia region of North America. Bull Univ Utah Biol Series 1944;35:1-267.

23. Furbee RB, Kao LW, Ibrahim D. Brown recluse spider envenomation. Clin Lab Med 2006;26:21126.

24. Isbister GK, Whyte IM. Suspected whitetail spider bite and necrotic ulcers. Intern Med J 2004;34:3844.

25. Benoit R, Suchard JR. Necrotic skin lesions: spider bite or something else? Consultant 2006;46:138694.

26. Isbister GK. Necrotic arachnidism: the myth of the modern plague. Lancet 2004;364:549-53. 